\title{
Pacific
}

Journal of

Mathematics

\section{SIMPLE GROUP ACTIONS ON HYPERBOLIC RIEMANN SURFACES OF LEAST AREA}

\section{S. AlLEN BRoughton}




\section{SIMPLE GROUP ACTIONS ON HYPERBOLIC RIEMANN SURFACES OF LEAST AREA}

\section{S. Allen Broughton}

It is shown that if a simple group $G$ acts conformally on a hyperbolic surface of least area (or alternatively, on a Riemann surface of least genus $\sigma \geq 2$ ), then $G$ is normal in $\operatorname{Aut}(S)$ and the map $\operatorname{Aut}(S) \rightarrow \operatorname{Aut}(G)$ induced by conjugation is injective. For the preponderance of these minimal actions the group $\operatorname{Aut}(S) / G$ is isomorphic to a subgroup of $\Sigma_{3}$. It is shown how to compute $\operatorname{Aut}(S)$ purely in terms of the group-theoretic structure of $G$, in these cases. As examples and as part of the proof, the minimal actions and the groups $\operatorname{Aut}(S)$ are completely worked out for $A_{5}, \mathrm{SL}_{3}(3), M_{11}$ and $M_{12}$.

1. Introduction. If $G$ is a finite group, then $G$ can act as a group of conformal (i.e. biholomorphic) automorphisms of a closed Riemann surface for infinitely many genera. Several authors, [C], [G-S1], [G-S2], [H1], [M], [T] and [W], have considered the question of determining the least genus of a surface on which a given group can act conformally. Tucker [T] calls this least genus the strong symmetric genus of the group, though we will adopt the terminology of H. Glover and call this least genus the action genus. Actions on such surfaces we shall call genus actions. Conder [C] has determined the action genera of all the alternating groups. Glover and Sjerve [G-S1], [G-S2] have determined the action genera for $\operatorname{PSL}_{2}\left(p^{k}\right), p$ a prime. Harvey [H1] and McLachlan [M] have worked out procedures to easily determine genus actions of cyclic and abelian groups, respectively.

If the surface $S$ has genus $\sigma \geq 2$, then $\operatorname{Aut}(S)$ is finite and, according to Hurwitz's famous theorem, $|\operatorname{Aut}(S)| \leq 84(\sigma-1)$. In this paper we consider the following question for simple groups (all our simple groups are non-abelian) acting on surfaces with genus $\geq 2$.

If the simple group $G$ acts conformally on the closed Riemann surface $S$, of least genus, then how large a subgroup of $\operatorname{Aut}(S)$ is $G$ ?

It turns out that $G$ is a normal subgroup of $\operatorname{Aut}(S)$ of very small index. This is our main result, Theorem 1.1 below. On the other hand for $e \geq 2$, and a prime $p>2 e+1$ there are genus actions of $G=\left(\mathbf{Z}_{p}\right)^{2 e}$ on surfaces $S$ of genus $\sigma=(e-1) p^{2 e}+1$ such that 
$N_{\text {Aut }(S)}(G) / G \simeq \operatorname{Aut}(S / G)$ and $S / G$ is conformally equivalent to a given surface of genus $e$ (see end of $\S 4$ for a proof sketch). Thus, the hypothesis of simplicity on $G$ leads to strong properties for genus actions of $G$. Incidentally, Greenberg [Gr] has proven that for any finite group $G$ there is a surface $S$ for which $G \simeq \operatorname{Aut}(S)$, but in general the genus of $S$ is much larger than the action genus of $G$. In [W], Woldar proves a theorem similar to Theorem 1.1, but only for simple groups which are images of triangle groups. Our Theorem 1.1 improves Woldar's result, and its proof uses quite different methods.

We are going to restrict our attention to surfaces of genus $\sigma \geq 2$ for reasons which we explain now. Any surface $S$ of genus $\sigma$ may be represented as a quotient $D / \Pi$ where $D$ is a simply-connected Riemann surface and $\Pi$ is a discontinuous, torsion-free subgroup of $\operatorname{Aut}(D)$, the group of biholomorphic transformations of $D$. There are 3 cases:

elliptic: $\quad \sigma=0, \quad D=S^{2}=$ Riemann sphere, $\operatorname{Aut}(D)=\operatorname{PSL}_{2}(\mathbf{C})$, parabolic: $\sigma=1, \quad D=\mathbf{C}, \operatorname{Aut}(D)=\left\{z \rightarrow a z+b: b \in \mathbf{C}, a \in \mathbf{C}^{*}\right\}$, hyperbolic: $\sigma \geq 2, \quad D=\mathbf{H}=$ upper half plane, $\operatorname{Aut}(D)=\operatorname{PSL}_{2}(\mathbf{R})$.

The finite groups of automorphisms of an elliptic Riemann surface are simply the finite subgroups of $\mathrm{PSL}_{2}(\mathbf{C})$, so the only action of a simple group that occurs is $A_{5}$, acting as the group of orientation-preserving symmetries of a regular icosahedron inscribed in a sphere (or the orientation-preserving symmetries of a soccer ball). For parabolic Riemann surfaces the only groups of automorphisms are finite quotients of the two dimensional orientation-preserving crystallographic groups and no simple group actions occur here (cf. [Z]). Thus, we restrict our attention to the hyperbolic case, i.e., $\sigma \geq 2$ and speak of hyperbolic action genus and hyperbolic genus actions. In the hyperbolic case, each biholomorphic automorphism in $\operatorname{Aut}(\mathbf{H})=\operatorname{PSL}_{2}(\mathbf{R})$ is also an isometry with respect to the hyperbolic Poincare metric on $\mathbf{H}$. Since $S=\mathbf{H} / \Pi, S$ also carries a metric for which the area of $S$ is $2 \pi(\sigma-1)$, if the invariant area form on $\mathbf{H}$ is taken to be $d x d y / y^{2}$. The action of $G$ on $S$ will also be isometric. In this terminology we have:

THeOREM 1.1. Let $G$ be a non-abelian simple group and let $S$ be a hyperbolic surface of least area on which $G$ acts conformally. Then $G \triangleleft \operatorname{Aut}(S)$ and the canonical map $\operatorname{Aut}(S) \rightarrow \operatorname{Aut}(G)$, induced by conjugation, is injective.

The proof is given in $\S 3$. The idea of the proof is to use topological 
considerations to show that any counterexample gives rise to a certain doubly transitive permutation representation of degree $<42$ of some simple group, satisfying some additional conditions. Using results of Conder [C], Sinkov [Sin], Sims [Si] and Liebeck and Saxl [L-S] we show this cannot happen. Our proof depends heavily on the following fact about simple groups, proven by Aschbacher and Guralnick [A-G].

Proposition 1.2. Every non-abelian simple group is generated by two elements.

A much simpler proof can be given for simple groups $G$ which satisfy:

Hypothesis 1.3. The non-abelian simple group $G$ is generated by an involution and another element.

According to Gorenstein [Go2] and Kantor [K] this generation hypothesis is very likely true for all simple groups though a formal proof has not been written down in every case. A large number of cases in which it is true are given in the paper by Aschbacher and Guralnick cited above. In [W], Woldar also makes heavy use of two-element generation of simple groups, in fact, he adopts Hypothesis 1.3 above for Theorem B in [W].

The classification theorem of simple groups is used in two ways to prove Theorem 1.1. First, the result of Aschbacher and Guralnick, Proposition 1.2, depends on the classification theorem; second, the results of Liebeck and Saxl [L-S] on primitive groups also depend on the classification theorem. If the Hypothesis 1.3 were assumed to be true, then it would not be necessary to use the results of Liebeck and Saxl (see Remark 3.1). The remainder of this paper is organized as follows. In $\S 2$ we recall some facts about conformal actions of groups on surfaces, show how to compute the number of genus actions and also how to compute $\operatorname{Aut}(S)$ for a wide class of genus actions. In $\S 3$ we prove Theorem 1.1. In $\S 4$ we work out some examples, $A_{5}, \mathrm{SL}_{3}(3)$, $M_{11}$ and $M_{12}$, in order to complete some details of the proof in $\S 3$ and to illustrate some of the ideas in $\S 2$. For contrast, we also work out, at the end of $\S 4$, the case of elementary abelian groups referred to above. In the paper we will use $\mathbf{Z}_{n}, \Sigma_{n}, A_{n}$ and $\mathbf{F}_{q}$ to denote the cyclic group of order $n$, the symmetric group and alternating groups on $n$ letters and the finite field of order $q$, respectively. All other notation is standard. 
2. Conformal group actions on surfaces. We recall some facts about conformal actions of groups and establish some notation. For background and any basic unreferenced results we use, we refer the reader to $[\mathbf{B e}],[\mathbf{F}-\mathbf{K}],[\mathbf{H} 2]$ and $[\mathbf{Z}]$. We say that the group $G$ acts conformally on the Riemann surface $S$ of genus $\sigma$ if there is given a monomorphism $\varepsilon: G \rightarrow \operatorname{Aut}(S)$, where $\operatorname{Aut}(S)$ is the group of biholomorphic transformations of $S$. If there will be no confusion we will simply consider $G$ as a subgroup of $\operatorname{Aut}(S)$.

We may represent $S$ as $D / \Pi, \Pi \subseteq \operatorname{Aut}(D)$ as described in $\S 1$. An element $x \in N_{\operatorname{Aut}(D)}(\Pi)$ induces an automorphism $\bar{x}$ of $S$, and every automorphism of $S$ arises in this way. For $H \subseteq \operatorname{Aut}(S)$ let $H^{*}=\left\{x \in N_{\operatorname{Aut}(D)}(\Pi): \bar{x} \in H\right\}$ and $G^{*}=(\varepsilon(G))^{*}$. We have:

$$
(1)^{*} \simeq \Pi, \quad(\operatorname{Aut}(S))^{*}=N_{\operatorname{Aut}(D)}(\Pi) \quad \text { and } \quad G \simeq G^{*} / \Pi .
$$

All our work depends on the following presentation of $G^{*}$ (cf. [Z]),

$$
\begin{aligned}
& G^{*}=\left\langle\alpha_{1}, \ldots, \alpha_{\tau}, \beta_{1}, \ldots, \beta_{\tau}, \gamma_{1}, \ldots, \gamma_{t}\right. \\
&\left.\prod_{i=1}^{\tau}\left[\alpha_{i}, \beta_{i}\right] \prod_{j=1}^{t} \gamma_{j}=\gamma_{1}^{n_{1}}=\cdots=\gamma_{t}^{n_{t}}=1\right\rangle .
\end{aligned}
$$

The integers $\tau$ and $n_{j}, j=1, \ldots, t$, have topological interpretations; in fact the presentation may be established by topological means. The quotient space $T=D / G^{*} \simeq S / G$ is a Riemann surface, its genus is $\tau$ which is $\leq \sigma$. The quotient map $q: D \rightarrow T$ is branched (ramified) over $t$ points $Q_{1}, \ldots, Q_{t} \in T$. The points $Q_{1}, \ldots, Q_{t}$ may be ordered so that for each $P \in q^{-1}\left(Q_{j}\right)$, the isotropy subgroup $G_{P}^{*}=\left\{x \in G^{*}: x P=P\right\}$ is conjugate to the cyclic subgroup of $G^{*}$ generated by $\gamma_{j}$. Also,

$$
o\left(\gamma_{j}\right)=n_{j} \text { in } G^{*}
$$

The integer $n_{j}$ is called the branching order at $Q_{j}$. Reorder $Q_{1}, \ldots$, $Q_{t}$, if necessary, so that $n_{1} \leq \cdots \leq n_{t}$. Let $B=\left\{\left(Q_{1}, n_{1}\right), \ldots,\left(Q_{t}, n_{t}\right)\right\}$ denote the branching set with branching orders. We call the $(t+1)$ tuple $\left(\tau: n_{1}, \ldots, n_{t}\right)$ the signature of $G^{*}$ or the branching data of $G$ acting on $S$. For convenience we write $\left(n_{1}, n_{2}, \ldots, n_{t}\right)$ instead of $\left(0: n_{1}, \ldots, n_{t}\right)$ since this type of branching data occurs so often.

The branching data and the order of $G$ are related by the Riemann- 
Hurwitz equation $[\mathbf{F}-\mathbf{K}],[\mathbf{Z}]$ :

$$
\frac{2 \sigma-2}{|G|}=2 \tau-2+\sum_{j=1}^{t}\left(1-\frac{1}{n_{j}}\right) \text {. }
$$

Define $\mu\left(G^{*}\right), \mu(G, S)$, and $\mu\left(\tau: n_{1}, \ldots, n_{t}\right)$ by:

$$
\mu\left(G^{*}\right)=\mu(G, S)=\mu\left(\tau: n_{1}, \ldots, n_{t}\right)=2 \tau-2+\sum_{j=1}^{t}\left(1-\frac{1}{n_{j}}\right) .
$$

According to (2.3) $S$ is elliptic $(\sigma=0)$, parabolic $(\sigma=1)$ or hyperbolic $(\sigma \geq 2)$ if and only if $\mu\left(G^{*}\right)<0, \mu\left(G^{*}\right)=0$ or $\mu\left(G^{*}\right)>$ 0 , respectively. We call the branching data $\left(\tau: n_{1}, \ldots, n_{t}\right)$ elliptic, parabolic or hyperbolic according to whether $\mu\left(\tau: n_{1}, \ldots, n_{t}\right)<0$, $=0$, or $>0$. From the Riemann-Hurwitz equation it immediately follows that if $H \subset G$ then,

$$
\mu(H, S)=[G: H] \mu(G, S) .
$$

Let $\xi: G^{*} \rightarrow \operatorname{Aut}(S)$ be the map induced by the action of $G^{*}$ on $D / \Pi$, the kernel of this map is $\Pi$. We may define $\eta: G^{*} \rightarrow G$ by $\eta=\varepsilon^{-1} \circ \xi$. Define the elements $a_{i}, b_{i}, c_{j}$ of $G$ by:

$$
\begin{aligned}
& a_{i}=\eta\left(\alpha_{i}\right), \quad 1 \leq i \leq \tau, \\
& b_{i}=\eta\left(\beta_{i}\right), \quad 1 \leq i \leq \tau, \\
& c_{j}=\eta\left(\gamma_{j}\right), \quad 1 \leq j \leq t .
\end{aligned}
$$

These elements generate $G$,

$$
\prod_{i=1}^{\tau}\left[a_{i}, b_{i}\right] \prod_{j=1}^{t} c_{j}=1
$$

and

$$
o\left(c_{j}\right)=n_{j},
$$

because of $(2.1),(2.2)$ and since $\Pi=\operatorname{ker}(\eta)$ is torsion free.

Definition 2.1. We call a $(2 \tau+t)$-tuple $\left(a_{1}, \ldots, a_{\tau}, b_{1}, \ldots, b_{\tau}\right.$, $\left.c_{1}, \ldots, c_{t}\right)$ of elements of $G$, satisfying (2.7)-(2.8), a $\left(\tau: n_{1}, \ldots, n_{t}\right)$ vector. Such a vector is called a generating $\left(\tau: n_{1}, \ldots, n_{t}\right)$-vector if $G=\left\langle a_{1}, \ldots, a_{\tau}, b_{1}, \ldots, b_{\tau}, c_{1}, \ldots, c_{t}\right\rangle$.

From the above we have Riemann's existence theorem (cf. [H1], $[\mathbf{T}])$. 
Proposition 2.1. The group $G$ acts conformally on a genus $\sigma$ surface $S$, with branching data $\left(\tau: n_{1}, \ldots, n_{t}\right)$ if and only if $2 \sigma-2=$ $|G| \mu\left(\tau: n_{1}, \ldots, n_{t}\right)$ and $G$ has a generating $\left(\tau: n_{1}, \ldots, n_{t}\right)$-vector.

REMARK 2.1. The (hyperbolic) action genus equals the minimum value of the quantity $1+|G| \mu\left(\tau: n_{1}, \ldots, n_{t}\right) / 2$ as we vary over all generating hyperbolic $\left(\tau: n_{1}, \ldots, n_{t}\right)$-vectors. For later use, we have recorded in Table 2.2, in increasing order, all the values of $\mu(G, S)$ satisfying $0<\mu(G, S) \leq 1 / 4$.

REMARK 2.2. As mentioned in the introduction, there are no parabolic actions of simple groups and the only elliptic action of a simple group is the $(2,3,5)$-action of $A_{5}$ on a sphere.

Equivalent Actions. If a group $G$ acts on a surface $S$ of genus $\sigma$, then there are two related questions to consider: (1) How many "inequivalent" actions are there? (2) What is the full automorphism group of $S$ ? First we define equivalent actions. If $\varepsilon^{\prime}: G \rightarrow \operatorname{Aut}\left(S^{\prime}\right)$ is a conformal action of $G$ on another surface, then we say that $\varepsilon$ and $\varepsilon^{\prime}$ are (conformally) equivalent actions if there is an $\omega \in \operatorname{Aut}(G)$ and conformal equivalence $h: S \rightarrow S^{\prime}$ such that

$$
\varepsilon^{\prime}(g)=h \varepsilon(\omega(g)) h^{-1} \text { for } g \in G .
$$

Let $\Pi^{\prime},\left(G^{*}\right)^{\prime}, T^{\prime}$ and $B^{\prime}$ denote the groups, surface and branch set, analogous to $\Pi, G^{*}, T$ and $B$, but defined with respect to the $G$-action on $S^{\prime}$. The two surfaces $S$ and $S^{\prime}$ will be conformally equivalent, via the conformal equivalence $h$ above, if and only if there is a $y \in \operatorname{Aut}(D)$ such that $\Pi^{\prime}=y \Pi y^{-1}$ and such that $h$ equals the induced map $\bar{y}: S \rightarrow S^{\prime}$ defined by $\bar{y}(\Pi x)=\Pi^{\prime} y x$. The $G$-actions on the two surfaces will be conformally equivalent if and only if this transformation $y$ satisfies $\left(G^{*}\right)^{\prime}=y G^{*} y^{-1}$. The transformation $y$ on $D$ induces a conformal equivalence $\hat{y}$ of $D / G^{*} \simeq S / G \simeq T$ to $D /\left(G^{*}\right)^{\prime} \simeq S /\left(G^{*}\right)^{\prime} \simeq T^{\prime}$, which maps branch points to branch points and preserves branching orders, i.e., $\hat{y}(B)=B^{\prime}$. On the other hand, if $k: D / G^{*} \rightarrow D /\left(G^{*}\right)^{\prime}$ is any conformal map, mapping branch points to branch points and preserving branching orders, then $k$ lifts to a covering map $y \in \operatorname{Aut}(D)$ such that $\left(G^{*}\right)^{\prime}=y G^{*} y^{-1}$ and $k=\hat{y}$. However, we do not necessarily have $\Pi^{\prime}=y \Pi y^{-1}$.

The conformal equivalence classes of $G$ actions with the same branching data may be classified by two sets of "invariants", one set consisting of continuous moduli, the other is a discrete set of invariants refining the moduli. Consider the set of all conformal equivalence 
classes of Riemann surfaces with a branch set $B$, where the location of the branch points are allowed to vary but not the branching orders, and where the conformal equivalences are supposed to map branch sets to branch sets, preserving branching orders. This set forms a complex algebraic variety called the moduli space. As a set, the moduli space may be also described as the set of conjugacy classes of discrete subgroups of $\operatorname{Aut}(D)$ with the presentation in (2.1). For the branching data $\left(n_{1}, n_{2}, n_{3}\right)$ and $\left(n_{1}, n_{2}, n_{3}, n_{4}\right)$ these moduli spaces are, respectively, a single point and the complex plane, possibly with some punctures. These are the cases most likely to occur for genus actions of simple groups. Woldar [W] has shown that for a genus action of a simple group satisfying Hypothesis $1.3, \tau=0$ and $t=3$ or 4 . By a similar argument, using Proposition 1.2, a genus action of any simple group must satisfy $\tau=0,3 \leq t \leq 5$ or $\tau=1, t=1$. Now, suppose we look at all actions of $G$ on $S$ with prescribed branching data and such that $G / S$ is conformally equivalent to a fixed surface $T$ with fixed branch set $B$ (we say that $S$ lies over $T$ with branch set $B)$. This corresponds to a single point in the moduli space, or a single conjugacy class of groups $G^{*}$. Therefore, every action of $G$ on a surface $S$ with $T \simeq S / G$ and branch set $B$ is determined by a homomorphism $\eta$ : $G^{*} \rightarrow G$ where $G^{*}$ is a fixed subgroup of $\operatorname{Aut}(D)$.

There may be several inequivalent actions corresponding to a point in the moduli space or, equivalently, the fixed group $G^{*}$ above. We can give an explicit description of the various classes with the help of the following definitions. Let $\mathscr{N}=N_{\text {Aut }(D)}\left(G^{*}\right)$. The group $\operatorname{Aut}(G) \times$ $\mathscr{N}$ acts on the set $\left\{\eta: G^{*} \rightarrow G, \eta\right.$ surjective, $\operatorname{ker}(\eta)$ torsion free $\}$ via the formula:

$$
\eta^{\prime}=\omega \circ \eta \circ \operatorname{Ad}_{y^{-1}}, \quad(\omega, y) \in \operatorname{Aut}(G) \times \mathscr{N},
$$

where $\operatorname{Ad}_{y^{-1}}(x)=y^{-1} x y$. By the formulae (2.6) we may transfer this action to the set of generating $\left(\tau: n_{1}, \ldots, n_{t}\right)$-vectors. The group $\operatorname{Aut}(G)$ acts freely on the set of generating $\left(\tau: n_{1}, \ldots, n_{t}\right)$-vectors, since an automorphism fixing the generators of a group must be trivial. Let $\mathscr{X}$ denote the orbit space of this action. The group $\operatorname{Aut}(G) \times \mathscr{N}$ acts on this set, clearly the kernel of this action contains $\operatorname{Aut}(G) \times G^{*}$. Thus we get an action of $\mathscr{N} / G^{*} \simeq(\operatorname{Aut}(G) \times \mathscr{N}) /\left(\operatorname{Aut}(G) \times G^{*}\right)$ on $\mathscr{X}$. Let $\operatorname{Aut}(T, B)$ denote the group of automorphisms of $T=D / G^{*}$ which preserves the branch set $B$. It is easy to prove that $\operatorname{Aut}(T, B)$ is naturally isomorphic to $\mathscr{N} / G^{*}$, and hence that it acts on $\mathscr{X}$. We have: 
Proposition 2.2. Let all notation be as immediately above. Then the equivalence classes of G-actions on surfaces $S$ lying over $T$ with branch set $B$ are in 1-1 correspondence with the $\operatorname{Aut}(T, B)$-orbits on $\mathscr{X}$. Moreover if $x_{0} \in \mathscr{X}$ corresponds to the surface $S$ and action $\varepsilon$ then $N_{\operatorname{Aut}(S)}(\varepsilon(G)) / \varepsilon(G)$ is canonically isomorphic to the stabilizer of $x_{0}$ under the $\operatorname{Aut}(T, B)$ action.

Proof sketch. The proposition may be easily proven by standard techniques; however since the author was unable to locate a direct statement in the literature we give a sketch of the proof. One may identify, in a 1-1 manner, the various equivalence classes of surfaces $S$ with $G$-action, lying over $T$ with branch set $B$, with the various kernels of surjective homomorphisms $\eta: G^{*} \rightarrow G$, where $G^{*}$ is held fixed. The action of $\operatorname{Aut}(T, B)$ on $\mathscr{X}$ may be identified with the action of $\mathscr{N}$ on these kernels. Once this identification is made the remainder of the proof is straightforward.

If the branching data is $\left(n_{1}, n_{2}, n_{3}\right)$, so that $G^{*}$ is a so-called triangle group, then the moduli space is a single point. Therefore, only the discrete invariants play a role. Since results in this case are simple to state and since most genus actions for simple groups are of this form, we give the complete result.

Proposition 2.3. Suppose that $G$ acts on the surface $S$ with branching data $\left(n_{1}, n_{2}, n_{3}\right)$. Then, by restriction of action, $\operatorname{Aut}(T, B)$ may be identified with the symmetry group of $B$, i.e., those permutations of $\left\{Q_{1}, Q_{2}, Q_{3}\right\}$ preserving the branching orders $\left\{n_{1}, n_{2}, n_{3}\right\}$. The action of $\operatorname{Aut}(T, B)$ on the space $\mathscr{X}$ of $\operatorname{Aut}(G)$-equivalence classes of generating $\left(n_{1}, n_{2}, n_{3}\right)$-vectors is given in Table 2.1 below.

TABLE 2.1

$\operatorname{Aut}(T, B)$-actions for branching data $\left(n_{1}, n_{2}, n_{3}\right)$

$\begin{array}{cccc}\left(n_{1}, n_{2}, n_{3}\right) & \operatorname{Aut}(T, B) & \text { Action on } T & \text { Effect on }\left(c_{1}, c_{2}, c_{3}\right) \\ n_{1}<n_{2}<n_{3} & \langle 1\rangle & \text { trivial } & \text { trivial } \\ n_{1}=n_{2}<n_{3} & \mathbf{Z}_{2}=\langle(1,2)\rangle & (1,2): 1-z & \left(c_{2}, c_{1}, c_{1}^{-1} c_{3} c_{1}\right) \\ n_{1}<n_{2}=n_{3} & \mathbf{Z}_{2}=\langle(2,3)\rangle & (2,3): z /(1-z) & \left(c_{3} c_{1} c_{3}^{-1}, c_{3}, c_{2}\right) \\ n_{1}=n_{2}=n_{3} & \Sigma_{3} & (1,2): 1-z & \left(c_{2}, c_{1}, c_{1}^{-1} c_{3} c_{1}\right) \\ & & (1,2,3): 1 /(1-z) & \left(c_{2}, c_{3}, c_{1}\right)\end{array}$


Notes on Table 2.1. (1) The branch points are assumed to be: $Q_{1}=$ $0, Q_{2}=1, Q_{3}=\infty$.

(2) In the third column $g: \phi(z)$ means that $g$ acts via the Möbius transformation $\phi(z)$.

Proof sketch. For the same reasons as in Proposition 2.2 we give a sketch of the proof. Since $\tau=0$, then $T$ is a sphere. The automorphism group of $T$ is sharply triply transitive which immediately establishes the identification of $\operatorname{Aut}(T, B)$ with the symmetry group of $B$. In Table 2.1 we have assumed that $Q_{1}=0, Q_{2}=1, Q_{3}=\infty$, and the elements of $\operatorname{Aut}(T, B)$ are given as explicit Möbius transformations. The action on generating vectors may be computed as follows. Let $T-B$ denote $T$ with punctures at the branch points in $B$. Pick a fourth point $Q_{0}$ in the triply punctured sphere $T-B$. Select three loops $\delta_{1}, \delta_{2}, \delta_{3} \in \pi_{1}\left(T-B, Q_{0}\right)$ such that $\delta_{j}$ loops once around $Q_{j}$ and $\delta_{1} \delta_{2} \delta_{3}$ is null-homotopic. Now let $D^{\circ}=q^{-1}(T-B)$ under the map $q: D \rightarrow D / G^{*}$. The group $G^{*}$ is the group of covering transformations of $D^{\circ} \rightarrow T-B$, and hence there is a map $\pi_{1}\left(T-B, Q_{0}\right) \rightarrow G^{*}$. The image of $\delta_{j}$ is $\gamma_{j}$ under this map. The action of an element $\operatorname{Aut}(T, B)$ on each $\delta_{j}$ may now be explicitly calculated, using the given Möbius transformations. Then, the action may be transferred to $c_{j}$ via $\gamma_{j}$. In some cases the computed action has been composed with an inner automorphism so that the resultant action has a simple form.

REMARK 2.3. If $n_{1}<n_{2}<n_{3}$ then:

$$
\# \text { genus }\left(n_{1}, n_{2}, n_{3}\right) \text {-actions }=\frac{\#\left(n_{1}, n_{2}, n_{3}\right) \text {-vectors }}{|\operatorname{Aut}(G)|} .
$$

REMARK 2.4. If the branching data is $\left(n_{1}, n_{2}, n_{3}, n_{4}\right)$, then $T$ is a sphere with four branch points. The structure of $\operatorname{group} \operatorname{Aut}(T, B)$ now depends on the modulus

$$
\lambda=\frac{\left(Q_{2}-Q_{3}\right)}{\left(Q_{2}-Q_{1}\right)} \frac{\left(Q_{4}-Q_{1}\right)}{\left(Q_{4}-Q_{1}\right)},
$$

as well as on the equality relations among the branching orders. The group is trivial for all but a finite set of moduli. The most complicated group occurs when $\lambda$ is a primitive sixth root of unity and the branching orders are all equal. The group is then isomorphic to $A_{4}$, realized as the orientation-preserving symmetries of a regular tetrahedron inscribed in a sphere. A table similar to Table 2.1 is not hard to construct, but it is somewhat complicated so we omit it for the sake of 
brevity. Moreover, the author knows of no genus action of a simple group that is branched over four points.

Homology Representation. (See also [Sc], [Sy].) If $G$ acts on $S$ then $G$ also acts on the homology group $H_{1}(S, \mathbf{C})$. This action, in particular the inequality (2.13) below, can be exploited in showing that certain types of actions do not exist. The action of $G$ on $S$ is uniquely determined by some generating $\left(\tau: n_{1}, \ldots, n_{t}\right)$-vector of $G$, say, $\left(a_{1}, \ldots, a_{\tau}, b_{1}, \ldots, b_{\tau}, c_{1}, \ldots, c_{t}\right)$. It is shown in [Br1] that the character $\eta$ of the homology representation on $H_{1}(S, \mathbf{C})$ is given by:

$$
\eta=2 \chi_{0}+(2 \tau-2+t) \rho-\sum_{j=1}^{t} \rho_{j},
$$

where $\chi_{0}$ is the principal character, $\rho$ is the character of the regular representation and $\rho_{j}$ for $j=1, \ldots, t$ is the permutation character of $G$ on the coset space $G /\left\langle c_{j}\right\rangle$. For any character $\chi$ and $g \in G$ let

$$
l_{g}(\chi)=\frac{1}{o(g)} \sum_{k=1}^{o(g)} \chi\left(g^{k}\right)
$$

it equals the multiplicity of the trivial character $\left.\chi_{0}\right|_{\langle g\rangle}$ in $\left.\chi\right|_{\langle g\rangle}$. By Frobenius reciprocity, it follows that if $\chi$ is a non-principal irreducible character of $G$, then the multiplicity of $\chi$ in $\eta$ is $(2 \tau-2+t) \chi(1)-\sum_{j=1}^{t} l_{c_{j}}(\chi)$. Thus,

$$
(2 \tau-2+t) \chi(1) \geq \sum_{j=1}^{t} l_{c_{j}}(\chi) .
$$

This inequality gives us a Brauer trick for proving that certain groups cannot act with a given branching data. In particular, to prove that the $\left(n_{1}, n_{2}, n_{3}\right)$-vector $\left(c_{1}, c_{2}, c_{3}\right)$ is not a generating vector, it suffices to find an irreducible non-trivial character $\theta$ such that:

$$
\theta(1)<l_{c_{1}}(\theta)+l_{c_{2}}(\theta)+l_{c_{3}}(\theta) \text {. }
$$

This inequality will be used in the proof of Proposition 3.9 and in some of the sample computations of genus actions in $\S 4$.

3. Proof of Theorem 1.1. We prove a series of propositions culminating in the proof of Theorem 1.1. Until further notice $G$ is a simple, non-abelian group acting conformally on a hyperbolic surface $S$ of least area and $R$ is any other hyperbolic surface on which $G$ acts. Let $\mu_{0}(G)=\mu(G, S)$, observe $\mu_{0}(G) \leq \mu(G, R)$. 
Proposition 3.1. If $G \simeq A_{5}$ then $\operatorname{Aut}(S) \simeq \Sigma_{5}$ and $G \triangleleft \operatorname{Aut}(S)$.

Proof. This calculation is done as Example 4.1.

Proposition 3.2. Let a simple group $G$ act on a hyperbolic surface $R$ and suppose $G$ is not normal in $\operatorname{Aut}(R)$. Then:

(i) If $\mu(G, R) \leq 1 / 4$ then $G$ is isomorphic to one of $A_{5}, A_{6}, A_{7}$, $A_{8}, A_{9}, \mathrm{PSL}_{2}(7)$ or $\mathrm{PSL}_{2}(8)$.

(ii) If $\mu(G, R)<1 / 6$ then $G \simeq A_{5}$.

Proof. Suppose first that $\mu(G, R) \leq 1 / 4$. Let $M=\operatorname{Aut}(R)$, by Table $2.2 \mu(M, R) \geq 1 / 42$ and $[M: G]=\mu(G, R) / \mu(M, R) \leq 42 / 4$. Consider the permutation representation of $M$ on $M / G$ restricted to $G$. At least one $G$-orbit is trivial, and since $G$ is simple every non-trivial orbit has at least 5 points. If $G$ is not normal then there is a non-trivial $G$-orbit of size $m, 5 \leq m \leq 9$. Since $G$ is simple we get a primitive permutation representation of $G$ whose degree is in the same range. From Sims' list of primitive groups of degree $\leq 20, G$ can only be one of the groups listed in (i). The proof of (ii) is similar.

Proposition 3.3. If $G \simeq A_{n}, n \geq 5$ or $G \simeq \operatorname{PSL}_{2}\left(p^{k}\right), p^{k} \geq 4$ or $G$ is a simple non-trivial permutation group of degree $\leq 20$, then $G \triangleleft \operatorname{Aut}(S)$.

Proof. $G \simeq A_{n}$. By Proposition 3.1, we may assume $n \geq 6$. Conder [C] has shown that any such alternating group has a genus action whose branching data is one of $(2,3,7),(2,3,8),(2,3,9),(2,3,10)$, $(2,3,11),(2,3,12),(2,4,5),(2,4,6),(2,4,7)$, or $(2,5,7)$. Thus $\mu_{0}\left(A_{n}\right)<1 / 6$ for $n \geq 6$ and we appeal to Proposition 3.2.

$G \simeq \operatorname{PSL}_{2}\left(p^{k}\right):$ If $p^{k}=5$ or 9 then $\mathrm{PSL}_{2}\left(p^{k}\right) \simeq A_{5}$ or $A_{6}$ which have already been considered. According to Sinkov [Sin] $\mathrm{PSL}_{2}\left(p^{k}\right)$ has a generating $(2,3, d)$-vector unless $p^{k} \leq 4$ or $p^{k}=9$. Since the only non-hyperbolic simple group action is the $(2,3,5)$-action of $A_{5}$, we may assume that $d \geq 7$. Thus, $\mu_{0}\left(\operatorname{PSL}_{2}\left(p^{k}\right)\right) \leq 1 / 6$, if $p^{k} \not \leq 5$ or $\neq 9$. Now argue as above.

$G$ is a permutation group. If $G$ is a simple permutation group of degree $\leq 20$, then it has a primitive permutation representation of degree $\leq 20$ and occurs in Sims' list. If we ignore those groups isomorphic to $\mathrm{PSL}_{2}\left(p^{k}\right)$ or $A_{n}$ we have only $\mathrm{SL}_{3}(3), M_{11}$, and $M_{12}$ to consider. In $\S 4$ we will compute $\mu_{0}\left(\mathrm{SL}_{3}(3)\right)=1 / 12, \mu\left(M_{11}\right)=$ 
$21 / 132, \mu\left(M_{12}\right)=1 / 15$; these cases then follow from Proposition 3.2 and the first two parts of this proposition.

Proposition 3.4. The index $[\operatorname{Aut}(S): G]<42$.

Proof. Because $G$ is simple, $G$ is generated by two elements $c_{1}, c_{2}$, according to Proposition 1.2. Let $c_{3}=\left(c_{1} c_{2}\right)^{-1}$ and $n_{j}=o\left(c_{j}\right)$; then $\left(c_{1}, c_{2}, c_{3}\right)$ is a generating $\left(n_{1}, n_{2}, n_{3}\right)$-vector. (If we do not have $n_{1} \leq n_{2} \leq n_{3}$ we may choose instead the $\left(n_{2}, n_{1}, n_{3}\right)$-vector $\left(c_{1} c_{2} c_{1}^{-1}, c_{1}, c_{3}\right)$ or the $\left(n_{3}, n_{1}, n_{2}\right)$-vector $\left(c_{2}, c_{3}, c_{1}\right)$ etc. $)$ By Proposition 2.1, $G$ acts on a surface $R$ with branching data $\left(n_{1}, n_{2}, n_{3}\right)$. This branching data cannot be elliptic or parabolic, according to Remark 2.2 , since we may safely assume $G \not A_{5}$. Thus $\mu_{0}(G)=$ $\mu(G, S)<\mu(G, R)=1-1 / n_{1}-1 / n_{2}-1 / n_{3}<1$. But now [Aut $(S): G$ ] $=\mu(G, S) / \mu(\operatorname{Aut}(S), S)<1.42$ since $\mu(\operatorname{Aut}(S), S) \geq 1 / 42$ by Table 2.2. More specifically we have:

\begin{tabular}{cc} 
Branching Data of $\operatorname{Aut}(S)$ on $S$ & {$[\operatorname{Aut}(S): G]$} \\
\hline$(2,3,7)$ & $<42$ \\
$(2,3,8)$ & $<24$ \\
everything else & $<20$
\end{tabular}

Proposition 3.5. If $G$ is not normal in $\operatorname{Aut}(S)$ then the permutation representation of $\operatorname{Aut}(S)$ on $\operatorname{Aut}(S) / G$ is doubly transitive of degree $n$ with $22 \leq n \leq 41$ and $\operatorname{Aut}(S) \not A_{n}$.

Proof. Let $M=\operatorname{Aut}(S)$ and assume that $G$ is not normal in $M$. Consider the permutation representation of $M$ on the coset space $M / G$, restricted to $G$. If there is more than one fixed point, then $G$ is strictly smaller than $N_{M}(G)$, and then the permutation representation of $G$ on the coset space $M / N_{M}(G)$ has an orbit of length $\leq 19$, because of Proposition 3.4. But now, by Proposition 3.3, $G \triangleleft \operatorname{Aut}(S)$, a contradiction. If there are more than two $G$-orbits or $[M: G] \leq$ 21, $G$ has a faithful permutation representation of degree $\leq 20$ and again Proposition 3.3 yields a contradiction. Thus $M$ acts doubly transitively and $22 \leq[M: G] \leq 41$. To see that $M \not A_{n}$ observe that $G \simeq A_{n-1}$ by order considerations and apply Proposition 3.3.

REMARK 3.1. If Hypothesis 1.3 is assumed then we may take $n_{1}=$ 2 in the proof of Proposition 4.1. Thus $\mu_{0}(G)<1 / 2$, implying 
TABLE 2.2

Branching data and $\mu\left(n_{1}, \ldots, n_{t}\right)$-values, $t=3,4, \mu \leq \frac{1}{4}$

\begin{tabular}{|c|c|c|c|c|c|}
\hline Branching Data & $\mu$ & Branching Data & $\mu$ & Branching Data & $\mu$ \\
\hline$(2,3,7)$ & $\frac{1}{42}$ & $(2,3, d), d \geq 133$ & $\frac{(d-6)}{6 d}$ & $(2,5,14)$ & $\frac{8}{35}$ \\
\hline$(2,3,8)$ & $\frac{1}{24}$ & $(2,4,12),(2,6,6),(3,3,6)$ & $\frac{1}{6}$ & $(2,4, d), 47 \leq d \leq 55$ & $\frac{(d-4)}{4 d}$ \\
\hline$(2,4,5)$ & $\frac{1}{20}$ & $(3,4,4),(2,2,3,3)$ & $\frac{1}{6}$ & $(2,4,56),(2,7,8)$ & $\frac{13}{56}$ \\
\hline$(2,3,9)$ & $\frac{1}{18}$ & $(2,4,13)$ & $\frac{9}{52}$ & $(2,4, d), 57 \leq d \leq 59$ & $\frac{(d-4)}{4 d}$ \\
\hline$(2,3, d), d=10,11$ & $\frac{(d-6)}{6 d}$ & $(2,5,8)$ & $\frac{7}{40}$ & $(2,4,60),(2,5,15)$ & $\frac{7}{30}$ \\
\hline$(2,3,12),(2,4,6),(3,3,4)$ & $\frac{1}{12}$ & $(2,4, d), 14 \leq d \leq 16$ & $\frac{(d-4)}{4 d}$ & $(3,6,10),(3,3,10)$ & $\frac{7}{30}$ \\
\hline$(2,3, d), d=13,14$ & $\frac{(d-6)}{6 d}$ & $(2,5,9)$ & $\frac{17}{90}$ & $(2,4, d), 61 \leq d \leq 79$ & $\frac{(d-4)}{4 d}$ \\
\hline$(2,3,15),(2,5,5)$ & $\frac{1}{10}$ & $(2,6,7),(3,3,7)$ & $\frac{4}{21}$ & $(2,4,80),(2,5,16)$ & $\frac{13}{56}$ \\
\hline$(2,3,16)$ & $\frac{5}{48}$ & $(2,4, d), 17 \leq d \leq 19$ & $\frac{(d-4)}{4 d}$ & $(2,4, d), 81 \leq d \leq 113$ & $\frac{(d-4)}{4 d}$ \\
\hline$(2,4,7)$ & $\frac{3}{28}$ & $(2,4,20),(2,5,10)$ & $\frac{1}{5}$ & $(2,5,17)$ & $\frac{41}{170}$ \\
\hline$(2,3,17)$ & $\frac{11}{102}$ & $(2,4, d), 21 \leq d \leq 23$ & $\frac{(d-4)}{4 d}$ & $(2,4, d), \quad 114 \leq d \leq 131$ & $\frac{(d-4)}{4 d}$ \\
\hline$(2,3, d), 18 \leq d \leq 23$ & $\frac{(d-6)}{6 d}$ & $(2,4,24),(2,6,8),(3,3,8)$ & $\frac{5}{24}$ & $(2,4,132),(2,6,11),(3,3,11)$ & $\frac{8}{33}$ \\
\hline$(2,3,24),(2,4,8)$ & $\frac{1}{8}$ & $(2,5,11)$ & $\frac{23}{110}$ & $(2,4, d), \quad 133 \leq d \leq 179$ & $\frac{(d-4)}{4 d}$ \\
\hline$(2,3, d), 25 \leq d \leq 29$ & $\frac{(d-6)}{6 d}$ & $(2,4, d), 25 \leq d \leq 27$ & $\frac{(d-4)}{4 d}$ & $(2,4,180),(2,5,18)$ & $\frac{11}{45}$ \\
\hline$(2,3,30),(2,5,6),(3,3,5)$ & $\frac{2}{15}$ & $(2,4,28),(2,7,7)$ & $\frac{3}{14}$ & $(2,4, d), \quad 181 \leq d \leq 251$ & $\frac{(d-4)}{4 d}$ \\
\hline$(2,3, d), 31 \leq d \leq 35$ & $\frac{(d-6)}{6 d}$ & $(2,4,29)$ & $\frac{25}{116}$ & $(2,4,252),(2,7,9)$ & $\frac{31}{126}$ \\
\hline$(2,3,36),(2,4,9)$ & $\frac{5}{36}$ & $(2,4,30),(2,5,12),(3,4,5)$ & $\frac{13}{60}$ & $(2,4, d), 253 \leq d \leq 379$ & $\frac{(d-4)}{4 d}$ \\
\hline$(2,3, d), 37 \leq d \leq 59$ & $\frac{(d-6)}{6 d}$ & $(2,4, d), 31 \leq d \leq 35$ & $\frac{(d-4)}{4 d}$ & $(2,4,380),(2,5,19)$ & $\frac{47}{190}$ \\
\hline$(2,3,60),(2,4,10)$ & $\frac{3}{20}$ & $(2,4,36),(2,6,9),(3,3,9)$ & $\frac{2}{9}$ & $(2,4, d), d \geq 381$ & $\frac{(d-4)}{4 d}$ \\
\hline$(2,3, d), 61 \leq d \leq 104$ & $\frac{(d-6)}{6 d}$ & $(2,4,37)$ & $\frac{33}{148}$ & $(2,5,20),(2,6,12),(2,8,8)$ & $\frac{1}{4}$ \\
\hline$(2,3,105),(2,5,7)$ & $\frac{11}{70}$ & $(2,5,13)$ & $\frac{29}{130}$ & $(3,3,12),(2,4,6),(4,4,4)$ & $\frac{1}{4}$ \\
\hline$(2,3, d), \quad 106 \leq d \leq 131$ & $\frac{(d-6)}{6 d}$ & $(2,4, d), 38 \leq d \leq 46$ & $\frac{(d-4)}{4 d}$ & $(2,2,2,4)$ & $\frac{1}{4}$ \\
\hline$(2,3,132),(2,4,11)$ & $\frac{21}{132}$ & & & & \\
\hline
\end{tabular}

[Aut $(S): G]<42 / 2=21$. Theorem 1.1 would now follow from the last proposition.

Proposition 3.6. Suppose that the permutation representation of $\operatorname{Aut}(S)$ on $\operatorname{Aut}(S) / G$ is primitive and $G$ is not normal in $\operatorname{Aut}(S)$, then $\operatorname{Aut}(S)$ is simple.

Proof. We may assume $G \not A_{5}$. Again let $M=\operatorname{Aut}(S)$ and let $N$ be a non-trivial normal subgroup of $M$. Since $G$ is simple, $N \cap G=$ 
$\langle 1\rangle$ or $G$. The group $M / N$ acts on $S / N$. If $G \cap N=\langle 1\rangle$ then $G$ acts effectively on $S / N$. If $G \not A_{5}$ then $S / N$ is hyperbolic by Remark 2.2, and from the Riemann-Hurwitz equation (2.3) it follows that $S / N$ has smaller genus than $S$, and hence smaller area than $S$. This contradiction shows that $G \cap N=G$. Since $G$ is a maximal subgroup and is not normal then $N=M$ and $M$ is simple.

Proposition 3.7. Suppose that $G$ is not normal in $\operatorname{Aut}(S)$; then $\operatorname{Aut}(S)$ is simple and one of the following holds.

(i) The branching data of $\operatorname{Aut}(S)$ on $S$ is $(2,3,7), n=[\operatorname{Aut}(S)$ : $G$ ] satisfies $22 \leq n \leq 41, \operatorname{Aut}(S) \neq A_{n}$ and $\operatorname{Aut}(S)$ acts doubly transitively on $\operatorname{Aut}(S) / G$.

(ii) The branching data of $\operatorname{Aut}(S)$ on $S$ is $(2,3,8), n=[\operatorname{Aut}(S)$ : $G]=22,23, \operatorname{Aut}(S) \not A_{n}$ and $\operatorname{Aut}(S)$ acts doubly transitively on $\operatorname{Aut}(S) / G$.

Proof. This is a direct consequence of the information in (3.1) and Propositions 3.5 and 3.6.

Proposition 3.8. Let $G, \operatorname{Aut}(S)$ be as in (i) or (ii) of Proposition 3.7. Then $\operatorname{Aut}(S)$ must be one of the groups listed in Table 3.1 below. In the table the index $[\operatorname{Aut}(S): G]$ and the order of an element of prime order of $G$ are given.

Proof. We use the classification results of Liebeck and Saxl [L-S] for primitive non-alternating groups with elements of large prime order. Their classification applies to those groups of degree $n=m p+k$ with an element of cycle type $1^{k} p^{m}, m<p, p$ a prime. For those with branching data $(2,3,7)$, we may take $p=7$ since $n<42=6.7$. For those with branching data $(2,3,8)$ we take $p=11$ or 23 and $n=22$ or 23 respectively. Then we simply run through the tables given in [L-S] looking for groups of degree $n$ for $22 \leq n \leq 41$ with an element of the correct prime order. In [L-S] the groups are classified by socle so we need only to consider Tables 2 and 3 of [L-S] where the socles are non-abelian simple. In each case $M$ will equal the listed socle. The work involved is a straightforward, tedious, numerical calculation, though the following remarks yield some shortcuts.

(a) For the $(2,3,8)$-cases we need only check Table 3 in [L-S] where the prime $p$ divides $n$.

(b) By construction the isotropy subgroups are isomorphic to the simple group $G$, thus we may ignore the cases in the tables where the 
TABLE 3.1

Case

$$
\operatorname{Aut}(S)
$$

$[\operatorname{Aut}(S): G] \quad$ Prime

$(2,3,7)$ branching data

$\begin{array}{ccc}M_{22} & 22 & 7 \\ M_{23} & 23 & 7 \\ M_{24} & 24 & 7 \\ {S p_{6}(2)}_{\mathrm{PSU}_{3}(3)} & 28 & 7 \\ \Omega_{6}^{+}(2) \simeq \mathrm{PSL}_{4}(2) \simeq A_{8} & 28 & 7 \\ \mathrm{PSL}_{2}(8) & 28 & 7 \\ A_{8} & 28 & 7 \\ A_{7} & 35 & 7 \\ \mathrm{PSL}_{4}(2) \simeq A_{8} & 36 & 7 \\ \mathrm{PSU}_{3}(3) & 36 & 7 \\ S_{6}(2) & 36 & 7 \\ A_{9} & 36 & 7 \\ (2,3,8) \text { branching data }_{22} & & \\ M_{22} & 22 & 11 \\ M_{23} & 23 & 23\end{array}$

isotropy subgroups are obviously not simple e.g., $\operatorname{PSL}_{d}(q)$ on the projective space of dimension $d-1$. Thus we eliminate $M=\operatorname{PSL}_{2}\left(p^{k}\right)$, $n=p^{k}+1$ and $M=\operatorname{PSL}_{5}(2), n=31$.

(c) In Table 3 , in the case where the socle is ${ }^{2} G_{2}(q)$, the relevant value of $q$ is 3 . But ${ }^{2} G_{2}(3)$ is of the form $\mathrm{Z}_{3} \ltimes S L_{2}(8)$ and so is not simple [Go1, p. 75]. This gives us case i.7.

Proposition 3.9. None of the cases in Proposition 3.7 gives rise to a counterexample to Theorem 1.1 .

Proof. It suffices to show that either there is not a generating vector of the required type or that the isotropy subgroup is not simple. 
To show that there are no generating $(2,3,7)$-vectors (generating $(2,3,8)$-vectors) we may either use Conder's results or just show that for each $(2,3,7)$-vector $((2,3,8)$-vector $)\left(c_{1}, c_{2}, c_{3}\right)$ there is an irreducible non-trivial character $\theta$ such that $(2.13)$ holds. Throughout this section and the next we use the notation $n_{m}$, respectively $n$, to denote the $m$ th $n$-dimensional character, respectively the unique $n$ dimensional character, occurring in a character table in [McK], where the group will be the current group under discussion. For instance, $l_{g}\left(12_{2}\right)$ would denote $l_{g}(\theta)$ where $\theta$ is the second twelve dimensional character from the designated character table.

Cases (i.1), (i.2), (i.3), (ii.1), (ii.2). $M=M_{22}, M_{23}, M_{24}$. From the character tables $M_{22}[\mathbf{M c K}], M_{23},\left[\mathbf{J}\right.$, p. 103] and $M_{24}[\mathbf{F r}, \mathrm{p}$. 346] we compute $l_{g}(\theta)$ for various $\theta$ and all $q$ of order $1,2,3$, 7 or $8\left(M_{24}\right.$ excepted $)$ and list them in tables below. The notation for conjugacy classes of elements is the cycle structure of elements in the degree 24 permutation representation in the last two tables and the standard notation in [McK] in the first table. By using (2.13) we see that $M_{22}$ and $M_{23}$ have no generating $(2,3,7)$ and $(2,3,8)$ vectors, and that $M_{24}$ has no generating $(2,3,7)$-vectors.

$$
\begin{aligned}
& \begin{array}{cccccccc}
M_{22} & g & 1 A & 2 A & 3 A & 7 A & 7 B & 8 A \\
\cline { 2 - 7 } & l_{g}(21) & 21 & 13 & 9 & 3 & 3 & 3
\end{array}
\end{aligned}
$$

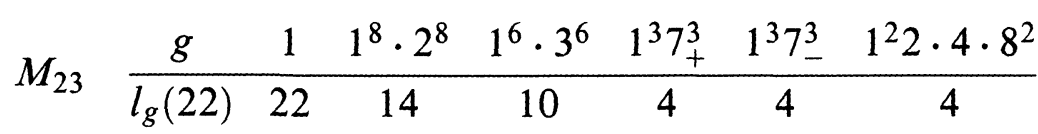

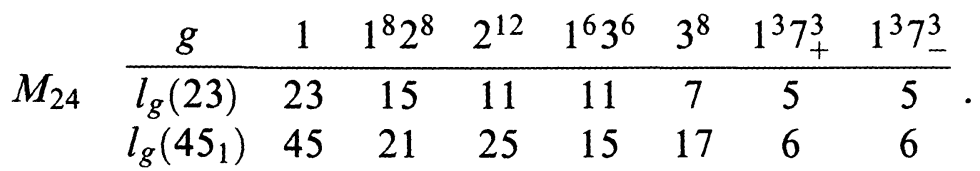

Cases (i.4), (i.12). $M=S p_{6}(2), n=28,36$. The isotropy subgroup has order $1,451,520 / 28=51,840$ or $1,451,520 / 36=$ 40,320 neither of which is the order of a simple group, according to the list in [McK].

Cases (i.5), (i.11). $M=\operatorname{PSU}_{3}(3), n=28,36$. From [McK] we form:

\begin{tabular}{ccccccc}
$g$ & $1 A$ & $2 A$ & $3 A$ & $3 B$ & $7 A$ & $7 B$ \\
\hline$l_{g}\left(21_{1}\right)$ & 21 & 13 & 9 & 7 & 3 & 3
\end{tabular}


There are no generating $(2,3,7)$-vectors.

Cases (i.6), (i.8), (i.9), (i.10), (i.13). $M \simeq \mathrm{PSL}_{4}(2)\left(\simeq A_{8}\right), A_{8}, A_{7}$, $\mathrm{PSL}_{4}(2)\left(\simeq A_{8}\right), A_{9}$. By Conder [C] none of these alternating groups have generating $(2,3,7)$-vectors.

Case (i.7). $M=\mathrm{PSL}_{2}(8)$. The isotropy subgroup has order 18 and is not simple.

We have now shown that for every simple group acting on a hyperbolic surface of least area that $G \triangleleft \operatorname{Aut}(S)$. To finish the proof of Theorem 1.1 we need to show that $\operatorname{Aut}(S) \rightarrow \operatorname{Aut}(G)$ induced by conjugation is injective. If this fails then there is $1 \neq g \in \operatorname{Aut}(S)$ which centralizes $G$. Since $G$ is centreless $\langle g\rangle \cap G=\langle 1\rangle$ and $\langle g\rangle \times G$ is a subgroup of $\operatorname{Aut}(S)$ hence acts on $S$. But then $G$ acts effectively on $S /\langle g\rangle$. If $G \not A_{5}$ then $S /\langle g\rangle$ is hyperbolic and $S /\langle g\rangle$ has smaller area by the Riemann Hurwitz equation, a contradiction. The remaining case $A_{5}$ is done in Example 4.1.

4. Examples. In this section we will determine all genus actions and work out $\operatorname{Aut}(S)$ for $A_{5}, \mathrm{SL}_{3}(3)$ and the two Mathieu groups, $M_{11}$ and $M_{12}$, and a class of elementary abelian groups. These examples were chosen to complete the proofs of Propositions 3.1 and 3.3, give a proof sketch for the unproven assertion on elementary abelian groups in $\S 1$, and to illustrate various techniques.

Simple Group Examples. The results for the simple groups are summarized in Table 4.1 (see next page). In the table we have recorded a result for $\operatorname{Aut}(S)$ only in those cases where $G$ is not the full automorphism group of $S$. The orders of the automorphism groups of the four groups considered are given in tables in [Go1]. Throughout this section we make extensive use of the tables in [McK] and [F-M] and we adopt the same notation for characters as in $\S 3$.

We next present two formulae that will allow us to calculate the number generating $\left(n_{1}, \ldots, n_{t}\right)$-vectors in a group. Let $K_{1}, \ldots, K_{t}$ be $t$ conjugacy classes in a finite group $G$, we call the $\left(n_{1}, \ldots, n_{t}\right)$ vector $\left(x_{1}, \ldots, x_{t}\right)$ a $\left(K_{1}, \ldots, K_{t}\right)$-vector if each $x_{j} \in K_{j}$. Let $X\left(K_{1}, \ldots, K_{t}\right)$ be the set of all $\left(K_{1}, \ldots, K_{t}\right)$-vectors. It is a well known result of character theory that the number of these vectors is 
TABle 4.1

Genus actions of sample simple groups

$\begin{array}{ccccc}G & \text { Branching Data } & \begin{array}{c}\text { Number of } \\ \text { Actions }\end{array} & \operatorname{Aut}(S) & \begin{array}{c}\text { Branching Data } \\ \text { of } \operatorname{Aut}(S)\end{array} \\ A_{5} & (2,5,5) & 1 & \Sigma_{5} & (2,4,5) \\ \mathrm{SL}_{3}(3) & (3,3,4) & 3 & \mathrm{Z}_{2} \ltimes \mathrm{SL}_{3}(3) & (2,3,8) \\ M_{11} & (2,4,11) & 2 & & \\ M_{12} & (2,3,10) & 1 & & \end{array}$

given by:

(4.1) $\left|X\left(K_{1}, \ldots, K_{t}\right)\right|$

$$
=\frac{|G|^{t-1}}{\left|\operatorname{Cent}\left(x_{1}\right)\right| \cdot \cdots \cdot\left|\operatorname{Cent}\left(x_{t}\right)\right|} \sum_{\chi} \frac{\chi\left(x_{1}\right) \cdots \cdot \chi\left(x_{t}\right)}{\chi(1)^{t-2}},
$$

where the sum is over the irreducible characters of $G$.

For a subgroup $L$ of $G$ let $X_{L}\left(K_{1}, \ldots, K_{t}\right)$ be the set of $\left(K_{1}, \ldots\right.$, $\left.K_{t}\right)$-vectors that generate $L$. For convenience we shall abbreviate $X_{L}\left(K_{1}, \ldots, K_{t}\right)$ to $X_{L}$ and $X\left(K_{1}, \ldots, K_{t}\right)$ to $X$. The number of generating $\left(K_{1}, \ldots, K_{t}\right)$-vectors is then given by:

$$
\begin{aligned}
\left|X_{G}\right| & =|X|-\sum_{L}\left|X_{L}\right|\left[G: N_{G}(L)\right] \\
& =|X|-|G| \sum_{L}\left(\left|X_{L}\right| /|L|\right)\left[N_{G}(L): L\right]^{-1},
\end{aligned}
$$

where $L$ runs over a set of representatives of conjugacy classes of proper subgroups of $G$ for which $X_{L}$ is non-empty.

EXAMPLE 4.1. $G=A_{5}$. Since $G$ only has elements of order 2, 3 and 5 , then from Table 2.2 , the least possible value of $\mu(G, S)$ is $1 / 10$ with branching data $(2,5,5)$. Every $(2,5,5)$-vector generates $G$ since the only proper subgroup of $G$ whose order is divisible by 10 is the dihedral group $D_{5}$ which has no $(2,5,5)$-vectors. In $A_{5}$ there is one conjugacy class of involutions $2 A$, and two classes of elements of order 5: $5 A, 5 B$. Using (4.1) we get $|X(2 A, 5 A, 5 A)|=$ $|X(2 A, 5 B, 5 B)|=0$ and $|X(2 A, 5 A, 5 B)|=|X(2 A, 5 B, 5 A)|=$ 60. Thus, there are $120=\left|\Sigma_{5}\right|=\left|\operatorname{Aut}\left(A_{5}\right)\right|(2,5,5)$-vectors. It follows that the set $\mathscr{X}$ in Proposition 2.2 consists of a single point. Thus there is only one hyperbolic genus action of $A_{5}$ and the hyperbolic action genus is 4 . From Table 2.2, $\operatorname{Aut}(T, B) \simeq \mathrm{Z}_{2}$, and acts on 
$G$ by $\left(c_{1}, c_{2}, c_{3}\right) \rightarrow\left(c_{3} c_{1} c_{3}^{-1}, c_{3}, c_{2}\right)$. The action is by outer automorphisms since it switches the two conjugacy classes $5 A, 5 B$. Thus $\operatorname{Aut}(S) \simeq \Sigma_{5}$. From Table 2.2 and equation (2.5) this action must be a $(2,4,5)$-action.

EXAMPLE 4.2. $G=\mathrm{SL}_{3}(3)$. From [McK] we compute:

\begin{tabular}{ccccccccccccc}
$g$ & $1 A$ & $2 A$ & $3 A$ & $3 B$ & $4 A$ & $6 A$ & $8 A$ & $8 B$ & $13 A$ & $13 C$ & $13 C$ & $13 D$ \\
\hline$l_{g}(12)$ & 12 & 8 & 6 & 4 & 4 & 4 & 2 & 2 & 0 & 0 & 0 & 0
\end{tabular}.

By applying (2.13) we conclude that there are no generating $(2,3, d),(2,4,6)$, or $(3,3,4)$-vectors except possibly $(2 A, 3 B$, $13 X)$-vectors, where $X$ is one of $A, B, C, D$, or $(3 B, 3 B, 4 A)$ vectors. By Table 2.2 we may ignore the $(2,3,13)$-vectors if we can find any generating $(3,3,4)$-vectors. We will show that there are three $\operatorname{Aut}(G)$-classes of generating $(3,3,4)$-vectors and that $\operatorname{Aut}(T, B)$ acts trivially on these classes. Using (4.1) we calculate that the number of $(3 B, 3 B, 4 A)$-vectors is $10|G|$.

Let $\left(c_{1}, c_{2}, c_{3}\right)$ be a $(3 B, 3 B, 4 A)$-vector and let $L=\left\langle c_{1}, c_{2}, c_{3}\right\rangle$. The subgroup $L$ is contained in a maximal subgroup whose order is divisible by 12 . From [F-M] this maximal subgroup must be one of $\Sigma_{4}, H_{1}$ or $H_{2}$ where the last two subgroups are defined by:

$$
\begin{aligned}
H_{1}=\left\{\left(\begin{array}{cc}
A & X \\
0 & a
\end{array}\right): A\right. & \in G L_{2}(3) \\
& \left.X=\left(\begin{array}{c}
x \\
y
\end{array}\right) x, y \in \mathbf{F}_{3}, a^{-1}=\operatorname{det}(A)\right\} .
\end{aligned}
$$

$$
H_{2}=\left\{g^{T}: g \in H_{1}\right\}, \quad T=\text { transpose } .
$$

The subgroup $\Sigma_{4}$ has no $(3,3,4)$-vectors because of the parity of the permutations involved. Thus $L$ is conjugate to a subgroup of $H_{1}$ or $H_{2}$. The group $\mathrm{SL}_{3}(3)$ has a single non-trivial outer automorphism $\lambda: g \rightarrow\left(g^{-1}\right)^{T}$ which interchanges $H_{1}$ and $H_{2}$. Therefore, without loss of generality, we may assume $L \subseteq H_{1}$, because $\lambda\left(H_{1}\right)=H_{2}$. Let $F_{1}=\mathrm{SL}_{2}(3) \ltimes \mathbf{F}_{3}^{2}$ be the subgroup of elements of $H_{1}$ with $\operatorname{det}(A)=1$ in (4.3.i). Since $\left(c_{1}, c_{2}, c_{3}\right)$ is a $(3,3,4)$-vector the images of $c_{1}, c_{2}$ and $c_{3}$ in $\mathrm{Z}_{2}=H_{1} / F_{1}$ must all be trivial and, hence, $L \subseteq F_{1}$.

We actually have $L=F_{1}$ as follows. Let $\phi: F_{1} \rightarrow \mathrm{SL}_{2}(3), g \rightarrow \bar{g}$ be the retraction onto the subgroup $\mathrm{SL}_{2}(3)$. Since $|\operatorname{ker}(\phi)|=9$ and $o\left(\bar{c}_{3}\right)=4$ it follows that $\left(\bar{c}_{1}, \bar{c}_{2}, \bar{c}_{3}\right)$ is a $(3,3,4)$-vector. The vector $\left(c_{1}, c_{2}, c_{3}\right)$ may be written $\left(\bar{c}_{1} v_{1}, \bar{c}_{2} v_{2}, \bar{c}_{3} v_{3}\right), v_{1}, v_{2}, v_{3} \in$ $V=\operatorname{ker}(\phi)$. Now the map $v \rightarrow\left[v, c_{3}\right]$ is an automorphism of 
$V$, since $c_{3}$ has order 4 , and acts without non-trivial fixed points on $V$. It follows that $\left\{v c_{3} v^{-1}=\left[v, c_{3}\right] c_{3}: v \in V\right\}$ is the coset $V c_{3}$. Conjugating by an element of $V$ we may transform our original generating vector into $\left(\bar{c}_{1} v_{4}, v_{5} \bar{c}_{2}, \bar{c}_{3}\right)$ for some $v_{4}, v_{5} \in V$. Since $c_{1} c_{2} c_{3}=1=\bar{c}_{1} \bar{c}_{2} \bar{c}_{3}=\bar{c}_{3} \bar{c}_{2} \bar{c}_{1}$, then conjugating by $\bar{c}_{1}^{-1}$, we get $1=\bar{c}_{1}^{-1} c_{1} c_{2} c_{3} \bar{c}_{1}=v_{4} v_{5} \bar{c}_{2} \bar{c}_{3} \bar{c}_{1}=v_{4} v_{5}$. Set $v=v_{4}$ so $v_{5}=v^{-1}$. Both $\bar{c}_{1} v$ and $v^{-1} \bar{c}_{2}$ have order 3 for any choice of $\bar{c}_{1}$ and $\bar{c}_{2}$, both of order 3 and any $v \in V$. Since $\bar{c}_{3}^{2}=\left(\begin{array}{cc}-1 & 0 \\ 0 & -1\end{array}\right)$ then $\bar{c}_{3}^{2} \bar{c}_{1} v \bar{c}_{3}^{-2}=\bar{c}_{1} v^{-1} \in L$ and, hence, $v^{2}=\left(\bar{c}_{1} v^{-1}\right)^{-1}\left(\bar{c}_{1} v\right) \in L$. Since $\bar{c}_{3}$ acts irreducibly on $\mathrm{F}_{3}^{2}$ then $L=F_{1}$ if and only if $v$ is not the identity element. An element of order 3 in $G$ lies in the conjugacy class $3 B$ if and only if the order of its centralizer is 9 which in turn occurs if and only if the minimal polynomial of the element has degree 3 . Therefore, $c_{1} v$ lies in $3 B$ if and only if $v$ does not lie in the 1-eigenspace of $c_{1}$ when this element is considered as a linear transformation of $V$. It follows that every $(3 B, 3 B, 4 A)$-vector, generating a subgroup of $H_{1}$, must generate all of $F_{1}$.

The intersection of the conjugacy class $3 B$ with $F_{1}$ is a disjoint union of two conjugacy classes $K_{B}^{+}$and $K_{B}^{-}$with representatives

$$
\left(\begin{array}{lll}
1 & 1 & 1 \\
0 & 1 & 1 \\
0 & 0 & 1
\end{array}\right) \text { and }\left(\begin{array}{ccc}
1 & -1 & 1 \\
0 & 1 & 1 \\
0 & 0 & 1
\end{array}\right) \text {, }
$$

respectively. The group $\mathrm{SL}_{2}(3)$ has a single conjugacy class of elements of order 4 . Thus, the elements of order 4 in $F_{1}$ form a single conjugacy class, namely $4 A=\bigcup_{g} g V$, where $g$ runs over the elements of order 4 in $\mathrm{SL}_{2}(3)$. We still denote this class by $4 A$. We may calculate the number of $(3 B, 3 B, 4 A)$-vectors in $F_{1}$ by using (4.1) to compute the number of $\left(K_{B}^{+}, K_{B}^{+}, 4 A\right),\left(K_{B}^{+}, K_{B}^{-}, 4 A\right),\left(K_{B}^{-}, K_{B}^{+}\right.$, $4 A)$ and $\left(K_{B}^{-}, K_{B}^{-}, 4 A\right)$-vectors in $F_{1}$. This calculation may be simplified by making use of the following observations. For any element $g$ in any finite group $G$ :

$$
\left|\operatorname{Cent}_{G}(g)\right|=\sum_{\chi \in \operatorname{Irr}(G)}|\chi(g)|^{2} .
$$

Also, for any element $g$ of order 4 in $F_{1}$,

$$
\left|\operatorname{Cent}_{F_{1}}(g)\right|=\left|\operatorname{Cent}_{\mathrm{SL}_{2}(3)}(\bar{g})\right|=4 \text {. }
$$

Therefore,

$$
\sum_{\chi \in \operatorname{Irr}\left(F_{1}\right)}|\chi(g)|^{2}=\sum_{\chi^{\prime} \in \operatorname{Irr}\left(\mathrm{SL}_{2}(3)\right)}\left|\chi^{\prime}(g)\right|^{2}
$$


But,

$$
\sum_{\chi \in \operatorname{Irr}\left(F_{1}\right)}|\chi(g)|^{2}=\sum_{\chi^{\prime}}\left|\chi^{\prime}(\bar{g})\right|^{2}+\sum_{\chi^{\prime \prime}}\left|\chi^{\prime \prime}(\bar{g})\right|^{2},
$$

where the $\chi^{\prime}$ are characters of irreducibles which factor through $\mathrm{SL}_{2}(3)$ and $\chi^{\prime \prime}$ runs though the remaining characters of $F 1$. The two equations above imply that $\chi^{\prime \prime}(g)=0$ for any element $g$, of order 4 , and all $\chi^{\prime \prime}$. Thus, in computing the right-hand side of (4.1) for the above types of vectors we get:

$$
\frac{\left|F_{1}\right|^{2}}{\left|\operatorname{Cent}\left(c_{1}\right)\right| \cdot\left|\operatorname{Cent}\left(c_{2}\right)\right| \cdot\left|\operatorname{Cent}\left(c_{3}\right)\right|} \sum_{\chi^{\prime} \in \operatorname{Irr}\left(\mathrm{SL}_{2}(3)\right)} \frac{\chi\left(\bar{c}_{1}\right) \chi\left(\bar{c}_{2}\right) \chi\left(\bar{c}_{3}\right)}{\chi(1)}
$$

where the centralizers are calculated in $F_{1}$. Using the character table of $\mathrm{SL}_{2}(3)[\mathrm{I}]$ we compute that,

$$
\left|X_{F_{1}}\left(K_{B}^{+}, K_{B}^{+}, 4 A\right)\right|=\left|X_{F_{1}}\left(K_{B}^{-}, K_{B}^{-}, 4 A\right)\right|=2\left|F_{1}\right|,
$$

and that there are no $\left(K_{B}^{+}, K_{B}^{-}, 4 A\right)$ or $\left(K_{B}^{-}, K_{B}^{+}, 4 A\right)$-vectors. Thus, both $F_{1}$ and $F_{2}$ have $4\left|F_{1}\right|$ generating $(3 B, 3 B, 4 A)$-vectors each. From equation (4.2) we get:

$$
\left|X_{G}\right|=10|G|-4\left|F_{1}\right| \cdot \frac{|G|}{2\left|F_{1}\right|}-4\left|F_{2}\right| \cdot \frac{|G|}{2\left|F_{2}\right|}=6|G|=3|\operatorname{Aut}(G)| \text {. }
$$

The set $\mathscr{X}$ of $\operatorname{Aut}(G)$-equivalence classes of generating vectors has three classes. Representatives of these classes, obtained by a computer search, are:

$$
\begin{array}{lll}
c_{1}=\left(\begin{array}{lll}
1 & 0 & 1 \\
2 & 0 & 0 \\
2 & 2 & 2
\end{array}\right), & c_{2}=\left(\begin{array}{lll}
1 & 0 & 0 \\
0 & 2 & 2 \\
2 & 1 & 0
\end{array}\right), & c_{3}=\left(\begin{array}{lll}
0 & 2 & 0 \\
1 & 0 & 0 \\
0 & 0 & 1
\end{array}\right), \\
c_{1}=\left(\begin{array}{lll}
1 & 0 & 0 \\
2 & 0 & 1 \\
1 & 2 & 2
\end{array}\right), & c_{2}=\left(\begin{array}{lll}
0 & 1 & 0 \\
1 & 0 & 2 \\
2 & 1 & 0
\end{array}\right), & c_{3}=\left(\begin{array}{lll}
0 & 2 & 0 \\
1 & 0 & 0 \\
0 & 0 & 1
\end{array}\right),
\end{array}
$$

and

$$
c_{1}=\left(\begin{array}{lll}
2 & 0 & 2 \\
1 & 0 & 2 \\
0 & 1 & 1
\end{array}\right), \quad c_{2}=\left(\begin{array}{lll}
1 & 1 & 0 \\
1 & 2 & 1 \\
2 & 1 & 0
\end{array}\right), \quad c_{3}=\left(\begin{array}{lll}
0 & 2 & 0 \\
1 & 0 & 0 \\
0 & 0 & 1
\end{array}\right) .
$$

From Table 2.1 $\operatorname{Aut}(T, B) \simeq \mathbf{Z}_{2}$. If we compose the transformation $\left(c_{1}, c_{2}, c_{3}\right) \rightarrow\left(c_{2}, c_{1}, c_{1}^{-1}, c_{3} c_{1}\right)$, given in the table, with conjugation by $c_{1}$ we obtain the transformation $\nu:\left(c_{1}, c_{2}, c_{3}\right) \rightarrow\left(c_{1} c_{2} c_{1}^{-1}, c_{1}, c_{3}\right)$, which induces the action of the non-trivial element of $\operatorname{Aut}(T, B)$ on 
classes of generating vectors. To show that $\operatorname{Aut}(T, B)$ acts trivially on $\mathscr{X}$ it suffices to show that for each of the three given vectors the $\nu$-image of a vector is equivalent to the original vector by an element of $\operatorname{Cent}_{\mathrm{Aut}\left(\mathrm{SL}_{3}(3)\right)}\left(c_{3}\right)$. This centralizer is generated by the outer automorphism $\lambda$ defined earlier and the inner automorphism $\operatorname{Ad}_{x}$ induced by the matrix:

$$
x=\left(\begin{array}{lll}
2 & 2 & 0 \\
1 & 2 & 0 \\
0 & 0 & 2
\end{array}\right)
$$

For these three vectors the elements to choose that induce the equivalence are, in order, $\operatorname{Ad}_{x^{6}} \circ \lambda, \operatorname{Ad}_{x^{6}} \circ \lambda$ and $\operatorname{Ad}_{x^{2}} \circ \lambda$, as may be verified by direct calculation.

EXAMPLE 4.3. $G=M_{11}$. From [McK] we have:

\begin{tabular}{ccccccccccc}
$g$ & $1 A$ & $2 A$ & $3 A$ & $4 A$ & $5 A$ & $6 A$ & $8 A$ & $8 B$ & $11 A$ & $11 B$ \\
\hline$l_{g}\left(10_{1}\right)$ & 10 & 6 & 4 & 4 & 2 & 2 & 2 & 2 & 0 & 0 \\
$l_{g}(11)$ & 11 & 7 & 5 & 3 & 3 & 3 & 1 & 1 & 1 & 1
\end{tabular}

By applying (2.13), we conclude that there are no generating $(2,3, d)$, $(2,4, d),(2,5,5),(2,5,6),(3,3,4)$ or $(3,3,5)$-vectors except possibly $(2,4,11)$-vectors. By Table 2.2 , if a $(2,4,11)$-action of $M_{11}$ exists it will be a genus action. By formula (4.1) the number of $(2,4,11)$-vectors is $2|G|$. Let $L$ be a subgroup generated by a $(2,4,11)$-vector. If $L$ is not all of $G$, then $L$ lies in a maximal subgroup whose order is divisible by 44 . From [F-M] the only maximal subgroup of $M_{11}$ divisible by 44 is $\operatorname{PSL}_{2}(11)$. Since the order of an element of $\mathrm{PSL}_{2}(11)$ divides 5,6 or 11 then, $\mathrm{PSL}_{2}(11)$ has no $(2,4,11)$-vectors. Therefore, $L=G$ and all $(2,4,11)$-vectors of $M_{11}$ are generating vectors. Since the outer automorphism group of $M_{11}$ is trivial, there are two inequivalent genus actions of $M_{11}$, by Remark 2.3.

EXAMPLE 4.4. $G=M_{12}$. We calculate from [McK] the following table:

\begin{tabular}{ccccccccc}
$g$ & $1 A$ & $2 A$ & $2 B$ & $3 A$ & $3 B$ & $4 A$ & $4 B$ & $5 A$ \\
\hline$l_{g}\left(11_{1}\right)$ & 11 & 5 & 7 & 5 & 3 & 5 & 3 & 3 \\
$l_{g}\left(11_{2}\right)$ & 11 & 5 & 7 & 5 & 3 & 3 & 5 & 3 \\
$l_{g}\left(16_{2}\right)$ & 16 & 10 & 8 & 4 & 6 & 4 & 2 & 4 \\
$l_{g}(66)$ & 66 & 36 & 34 & 24 & 22 & 16 & 16 & 14
\end{tabular}




\begin{tabular}{cccccccc}
$g$ & $6 A$ & $6 B$ & $8 A$ & $8 B$ & $10 A$ & $11 A$ & $11 B$ \\
\hline$l_{g}\left(11_{1}\right)$ & 1 & 3 & 3 & 1 & 1 & 1 & 1 \\
$l_{g}\left(11_{2}\right)$ & 1 & 3 & 1 & 3 & 1 & 1 & 1 \\
$l_{g}\left(16_{2}\right)$ & 4 & 2 & 2 & 1 & 2 & 1 & 1 \\
$l_{g}(66)$ & 12 & 12 & 8 & 8 & 8 & 6 & 6
\end{tabular}

Using (2.13), there cannot be any generating $(2,3,8),(2,4,5)$, $(2 A, 3 A, 10 A),(2 A, 3 B, 10 A)$ or $(2 B, 3 A, 10 A)$-vectors. If a generating $(2 B, 3 B, 10 A)$-vector exists then all genus actions will be $(2 B, 3 B, 10 A)$-actions, by Table 2.2. By calculation, the number of $(2 B, 3 B, 10 A)$-vectors is $2|G|=\left|\operatorname{Aut}\left(M_{12}\right)\right|$. Let $\left(c_{1}, c_{2}, c_{3}\right)$ be a $(2 B, 3 B, 10 A)$-vector which is not a generating vector and let $L$ be the proper subgroup generated by this vector. Since $L$ must lie in a maximal subgroup whose order is divisible by $60, L$ lies in a subgroup isomorphic to one of $\mathrm{Z}_{2} \times \Sigma_{5}, \mathrm{PSL}_{2}(11), \mathrm{Z}_{2} \times M_{10}$ or $M_{11}$, according to the tables in [F-M]. The two possibilities $\operatorname{PSL}_{2}(11)$ and $M_{11}$ can be rejected since these subgroups do not have elements of order 10 , according to the tables in [McK].

Now let us eliminate $\mathbf{Z}_{2} \ltimes M_{10}$. This subgroup has a normal subgroup of index 4 isomorphic to $A_{6} \simeq \mathrm{PSL}_{2}(9)$. Since $c_{2}$ has odd order it must lie in $A_{6}$. By restricting characters of $M_{12}$ to $A_{6}$ we will show that $c_{2}$ lies in the conjugacy class $3 A$ of $M_{12}$, a contradiction. The restriction of any character $\theta$ of $M_{12}$ to $A_{6}$ must be an $\operatorname{Aut}\left(A_{6}\right)$ invariant character since $\operatorname{Aut}\left(A_{6}\right) \simeq \mathbf{Z}_{2} \ltimes M_{10}$ is an intermediate subgroup. Suppose that the dimension of $\theta$ is 11 . Then, using invariance and dimension considerations, we see that the only possible decompositions for the restricted character $\left.\theta\right|_{A_{6}}$ are $1+5_{1}+5_{2}, 2 \cdot 1+9,1+10$ or $11 \cdot 1$. Since the value of an 11-dimensional character on an element of order 5 equals 1 , then the case where $\theta$ restricts to the trivial representation with character $11 \cdot 1$ must be excluded. In each of the remaining cases the value of the restricted character at an element of order 3 in $A_{6}$ is 2 . But from the character table of $M_{12}$ this can only happen if the element of order 3 lies in the conjugacy class $3 A$, yielding a contradiction.

Finally we eliminate $\mathbf{Z}_{2} \times \Sigma_{5}$ in a similar fashion. In $\mathbf{Z}_{2} \times \Sigma_{5}$ let $\mathbf{Z}_{2}=\langle x\rangle$. Suppose that the $(2 B, 3 B, 10 A)$-vector $\left(c_{1}, c_{2}, c_{3}\right)$ lies in $\mathbf{Z}_{2} \times \Sigma_{5}$, then $\left(c_{1}, c_{2}, c_{3}\right)=\left(x b_{1}, b_{2}, x b_{3}\right)$ where $\left(b_{1}, b_{2}, b_{3}\right)$ is a generating $(2,3,5)$-vector of $A_{5}$. The elements $b_{1}$ and $b_{2}$ lie in the classes $2 B$ and $3 B$ of $M_{12}$, respectively. For $b_{2}=c_{2}$ and $b_{1}$ is the square of an element of order 4 , so, from the squaring maps in McKay's tables, $b_{1}$ lies in the conjugacy class $2 B$. If $\theta$ is the 
character above, then the dimension of $\theta$ is 11 and $\theta$ has the value $3,-1$ and 1 on the elements of order 2,3 and 5 in $A_{5}$, respectively. This information is sufficient to determine the restricted character $\left.\theta\right|_{A_{5}}$ uniquely, we get $\left.\theta\right|_{A_{5}}=1+2 \cdot 5$. The order of $\operatorname{Cent}(x)$ is divisible by $240=\left|\mathbf{Z}_{2} \times \Sigma_{5}\right|$, so $x$ lies in the class $2 A$ and $\theta$ has the value -1 on $x$. Since $x$ centralizes $A_{5}$ then $x$ acts as 1 or -1 on the irreducible constituents of $\left.\theta\right|_{A_{5}}$. Since the traces of $x$ on these subspaces must add up to -1 , these traces can only be $-1,5$ and -5 . It now follows from the character table of $A_{5}$ that $x b_{1}$ will have the traces $-1,1,-1$ on these subspaces and hence $\theta\left(c_{1}\right)=-1$. Thus $c_{1}$ lies in the conjugacy class $2 A$, a contradiction. All is now proven.

Elementary Abelian Groups. As supposed in the example in the introduction, let $e \geq 2$, let $p \geq 2 e+1$ be a prime, let $G=\left(\mathbf{Z}_{p}\right)^{2 e}$. Also let $\sigma_{e}=(e-1) p^{2 e}+1$; note that $\left(2 \sigma_{e}-2\right) / p^{2 e}=2 e-2$. The group $G$ clearly has a generating $(e:-)$-vector $\left(a_{1}, \ldots, a_{e}, b_{1}, \ldots, b_{e}\right)$ and so acts on a surface of genus $\sigma_{e}$. In [Br2] it is shown that for any prime $p$ and exponent $\beta$ there is a $\left(\tau: n_{1}, \ldots, n_{t}\right)$-vector $\left(a_{1}, \ldots, a_{\tau}\right.$, $\left.b_{1}, \ldots, b_{\tau}, c_{1}, \ldots, c_{t}\right)$ of $\left(\mathbf{Z}_{p}\right)^{\beta}$, if and only if the following hold:

$$
\begin{gathered}
t, \tau \geq 0, \quad t \neq 1, \\
2 \sigma-2=p^{\beta}[(2 \tau-2+t)-t / p], \\
\beta \leq 2 \tau \quad \text { if } t=0, \quad \beta<2 \tau+t \quad \text { if } t>1 .
\end{gathered}
$$

Setting $\beta=2 e$ and supposing $t>0$ we have $2 \tau+t=2 e+l$, where $l \geq 1$ and $t \leq 2 e+l$. Let $\sigma$ denote the genus of the surface on which $G$ acts via the generating vector $\left(a_{1}, \ldots, a_{\tau}, b_{1}, \ldots, b_{\tau}, c_{1}, \ldots, c_{t}\right)$. Then,

$$
\begin{aligned}
\frac{(2 \sigma-2)}{p^{2 e}} & =2 \tau-2+t-t / p \\
& =2 e-2+l-t / p \\
& \geq 2 e-2+l-(2 e+l) / p \\
& =2 e-2+l(1-1 / p)-2 e / p \\
& \geq 2 e-2+(1-1 / p)-2 e / p \\
& =2 e-2+(1-(2 e+1) / p) \\
& >\frac{\left(2 \sigma_{e}-2\right)}{p^{2 e}} .
\end{aligned}
$$

It follows that all genus actions of $G$ are given by $(e:-)$-vectors. It is also clear that the $(e:-)$-vectors, which are simply vector space 
bases of $G$ are $\operatorname{Aut}(G)$-equivalent, so there is only one genus action.

Now suppose that $T$ is a surface of genus $e$ and $H=\operatorname{Aut}(T)$. There are many different possibilities for $H$ obtained by varying the conformal structure of $T$. See [Br3] for a classification for $e=2,3$. Let $S \rightarrow T$ be the covering space determined by the kernel of the map $\Pi_{1}(T) \rightarrow H_{1}(T ; \mathbf{Z}) \rightarrow\left(\mathbf{Z}_{p}\right)^{2 e}$ where the last map is reduction $\bmod p$. This is a regular covering space whose group of covering transformations is isomorphic to $G$. The resulting $G$-action is a genus action, and every genus action can be derived in exactly this way. Via the covering projection $S \rightarrow T$ one may pull back the conformal structure on $T$ to a conformal structure on $S$, such that $G$ acts conformally. Let $M=N_{\operatorname{Aut}(S)}(G)$, then covering space arguments may be used to show that the natural map $M \rightarrow \operatorname{Aut}(S / G)=\operatorname{Aut}(T)$ is surjective. It would be interesting to see if there are any cases where $M$ is a proper subgroup of $\operatorname{Aut}(S)$.

\section{REFERENCES}

[A-G] M. Aschbacher and R. Guralnick, Some applications of the first cohomology group, J. Algebra, 90 (1984), 446-460.

[Be] A. F. Beardon, Geometry of Discrete Groups, Graduate Texts in Mathematics, Springer Verlag, Berlin and New York.

[Br1] A. Broughton, The homology and higher representations of the automorphism group of a Riemann surface, Trans. Amer. Math. Soc., 300, No. 1 (1987), 153-158.

[Br2] _ The equisymmetric stratification of the moduli space and the Krull dimension of mapping class groups, Topology Appl., 37 (1990), 101-113.

[Br3] _ Classifying finite group actions on surfaces of low genus, J. Pure Appl. Algebra, 69 (1990), 233-270.

[C] M. Conder, The symmetric genus of alternating and symmetric groups, J. Combin. Theory Ser. B, 39 (1985), 179-186.

[F-K] H. Farkas and I. Kra, Riemann Surfaces, Graduate Texts in Math., No. 71, Springer-Verlag, Berlin and New York (1980).

[F-M] J. Fischer and J. McKay, The non-abelian simple groups $G,|G|<10^{6}-$ maximal subgroups, Math. Comp., 32, No. 144 (1978), 1293-1302.

[Fr] G. Frobenius, Gesammelte Abhandlungen, Vol. 3, Springer-Verlag, Berlin and New York (1968).

[G-S1] H. Glover and D. Sjerve, Representing $\mathrm{PSL}_{2}(p)$ on a surface of least genus, L'Enseignement Mathématique, 31 (1985), 305-325.

[G-S2] _ The genus of $\operatorname{PSL}_{2}(q)$, J. Reine Angew. Math., 380 (1987), 59-86.

[Go1] D. Gorenstein, Finite Simple Groups, Plenum (1982).

[Go2] _ personal communication.

[Gr1] L. Greenberg, Maximal Fuchsian groups, Bull. Amer. Math. Soc., 69 (1963), 569-573.

[H1] J. Harvey, Cyclic groups of automorphisms of compact Riemann surfaces, Quarterly J. of Math. (Oxford Ser. 2), 17 (1966), 86-97. 
[H2] _ On branch loci in Teichmüller space, Trans. Amer. Math. Soc., 153 (1971), 387-399.

[I] I. M. Isaacs, Character Theory of Finite Groups, Academic Press (1976).

[J] G. D. James, The modular characters of the Mathieu groups, J. Algebra, 27 (1973), 57-111.

[K] W. Kantor, personal communication.

[L-S] M. Liebeck and J. Saxl, Primitive permutation groups containing an element of large prime order, J. London Math. Soc. (2), 31 (1985), 237-249.

[McK] J. McKay, The non-abelian simple groups $G,|G|<10^{6}$-character tables, Comm. Algebra, 7, No. 13 (1979), 1407-1445.

[M] C. Maclachlan, Abelian groups of automorphisms of compact Riemann surfaces, Proc. London Math. Soc., Ser. 3, 15 (1965), 699-712.

[Sc] L. Scott, Matrices and cohomology, Ann. of Math., 105 (1977), 473-492.

[Si] C. Sims, Computational methods in the study of permutation groups, in Computational Problems in Abstract Algebra (Proc. Conf. Oxford (1967), J. Leech (ed.)), Pergamon, Oxford (1970), 169-173.

[Sin] A. Sinkov, On the group defining relations $(2,3,7 ; \rho)$, Ann. Math. (Ser. 2), 38 (1937), 577-584.

[Sy] P. Symonds, The cohomology representation of an action of $C_{p}$ on a surface, Trans. Amer. Math. Soc., 306, No. 1 (1988), 389-400.

[T] T. Tucker, Finite groups acting on surfaces and the genus of a group, J. Combin. Theory Ser. B, 34 (1983), 82-98.

[W] A. Woldar, Genus actions of finite simple groups, preprint, Villanova University.

[Z] H. Zieschang, Finite Groups of Mapping Classes of Surfaces, Lecture Notes in Math., No. 875, Springer-Verlag, Berlin, New York (1981).

Received February 1, 1990 and in revised form, December 20, 1991.

Cleveland State University

Cleveland, $\mathrm{OH} 44115$ 


\title{
PACIFIC JOURNAL OF MATHEMATICS
}

Founded by

\author{
E. F. BeCKeNBACH (1906-1982) F. Wolf (1904-1989)
}

\section{EDITORS}

\section{S. VARADARAJAN (Managing Editor) University of California Los Angeles, CA 90024-1555 vsv@math.ucla.edu \\ F. Michael Christ University of California Los Angeles, CA 90024-1555 christ@math.ucla.edu}

\section{Herbert Clemens}

University of Utah

Salt Lake City, UT 84112

clemens@math.utah.edu

\author{
THOMAS ENRIGHT \\ University of California, San Diego \\ La Jolla, CA 92093 \\ tenright@ucsd.edu \\ Nicholas ERcolani \\ University of Arizona \\ Tucson, AZ 85721 \\ ercolani@math.arizona.edu \\ R. FINN \\ Stanford University \\ Stanford, CA 94305 \\ finn@gauss.stanford.edu \\ VAUGHAN F. R. JONES \\ University of California \\ Berkeley, CA 94720 \\ vfr@math.berkeley.edu
}

\section{SUPPORTING INSTITUTIONS}

UNIVERSITY OF ARIZONA

UNIVERSITY OF BRITISH COLUMBIA

UNIVERSITY OF OREGON

CALIFORNIA INSTITUTE OF TECHNOLOGY

UNIVERSITY OF CALIFORNIA

UNIVERSITY OF MONTANA

UNIVERSITY OF NEVADA, RENO

NEW MEXICO STATE UNIVERSITY

OREGON STATE UNIVERSITY
SteVen KerckhofF

Stanford University

Stanford, CA 94305

spk@gauss.stanford.edu

MARTIN SCHARLEMANN

University of California

Santa Barbara, CA 93106

mgscharl@henri.ucsb.edu

HAROLD STARK

University of California, San Diego

La Jolla, CA 92093

\author{
STANFORD UNIVERSITY \\ UNIVERSITY OF HAWAII \\ UNIVERSITY OF UTAH \\ WASHINGTON STATE UNIVERSITY \\ UNIVERSITY OF WASHINGTON
}

UIV 


\section{PACIFIC JOURNAL OF MATHEMATICS}

Volume $158 \quad$ No. $1 \quad$ March 1993

Determinant identities

GEORGE W. Eyre ANDrEWS and WiLliam H. Burge

A spectral theory for solvable Lie algebras of operators

E. BOASSO and ANGEL RAFAEL LAROTONDA

Simple group actions on hyperbolic Riemann surfaces of least area

S. Allen Broughton

Duality for finite bipartite graphs (with an application to $\mathrm{II}_{1}$ factors)

MARIE CHODA

Szegő maps and highest weight representations

MARK GREGORY DAVIDSON and Ron STANKE

Optimal approximation class for multivariate Bernstein operators

ZEEV DitZIAN and XINLONG ZHOU

Witt rings under odd degree extensions

ROBERT FITZGERALD

Congruence properties of functions related to the partition function

ANTHONY D. FORBES

Bilinear operators on $L^{\infty}(G)$ of locally compact groups

Colin C. GRAham and Anthony To-Ming LAU

Nonuniqueness of the metric in Lorentzian manifolds

GEOFFREY K. MARTIN and GERARD THOMPSON

Index theory and Toeplitz algebras on one-parameter subgroups of Lie 189 groups

EFTON PARK 